\section{Support for the involvement of complement factor I in age-related macular degeneration}

European Journal of Human Genetics (2010) 18, 15-16; doi:10.1038/ejhg.2009.113; published online 15 July 2009

We read with interest the article published in the January 2009 issue of this journal entitled 'Variation near complement factor I is associated

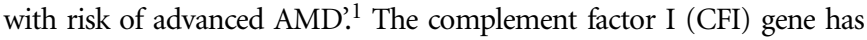
been the focus of study within our own UK cohort of age-related macular degeneration patients and controls. Soon after the publication of the seminal papers implicating the complement factor $\mathrm{H}(\mathrm{CFH})$ gene, ${ }^{2-4}$ we conducted a case-control study of this gene involving 190 DNA samples from patients and 179 control samples. This cohort has been earlier described. ${ }^{5}$ Briefly, all participants were white, aged more than 55 years, and ascertained through ophthalmic research clinics in the Southampton locality. All participants provided informed consent and were examined by an experienced retinal specialist. All patients had an AREDS classification of 2 or greater. Control samples were either spouses or partners of patients with disease or those who presented at the eye clinics for an unrelated eye disease. Summary demographics of the cohort used are detailed in Table 1.

After correction for multiple testing, our results were of marginal significance and so of somewhat unclear relevance to AMD when appraised in isolation. However, in light of results published by Fagerness et al in a larger sample (1228 cases and 825 controls), the data are of renewed interest and are presented here in support of their recent finding.

We genotyped six SNPs in the CFI region (Figure 1), four of which were also genotyped by Fagerness et al including their most significant SNP rs10033900 and its partner in the most significant haplotype rs13117504. Genotyping was carried out by KBioscience (Hoddesdon, Hertfordshire, UK) using KASPar chemistry (http://www.kbioscience. co.uk/genotyping/genotyping_chemistry.html).

Data were analysed using PLINK software (Version 1.06, http://pngu.mgh.harvard.edu/purcell/plink/). ${ }^{7}$ A high genotyping rate $(>98.6 \%)$ was achieved and all SNPs conformed to HardyWeinberg equilibrium as tested in the control samples only. Results of

Table 1 Demographics of case-control cohort

AREDS classification for cases only (n)

\begin{tabular}{lcccccc} 
& Male/female & Mean age (SD) & 1 & 2 & 3 & 4 \\
\hline Cases & 0.49 & $76.9(8.4)$ & 0 & 12 & 21 & 157 \\
Controls & 0.95 & $77.2(7.1)$ & & & & \\
\hline
\end{tabular}

our single SNP analysis are provided in Table 2. Although four SNPs are nominally significant in this initial analysis, no single SNP maintains marginal significance after (conservative) Bonferroni correction for multiple testing. Furthermore, these four SNPs have very similar minor allele frequencies and are likely to be genetically hitchhiking with one another. Of the four SNPs common to both studies, two SNPs found to be significant in the US study are not formally significant in our sample. However, it is interesting that rs11726949 has a suggestive $P$-value $(0.09)$ and a very similar odds ratio $(\mathrm{OR})$ in both studies $\left(\mathrm{OR}_{\mathrm{US}}=1.63, \mathrm{OR}_{\mathrm{UK}}=1.64\right)$. The other two SNPs common to both studies (rs6854876 and rs13117504) show independent significance across samples with highly concordant estimates of OR.

Using the Haploview software (version 4.1, http://www.broad.mit. $\mathrm{edu} / \mathrm{mpg} /$ haploview/ $/)^{6}$ and the solid spine of LD method $\left(D^{\prime}>0.8\right)$, we estimated haplotype block boundaries in our sample and analysed
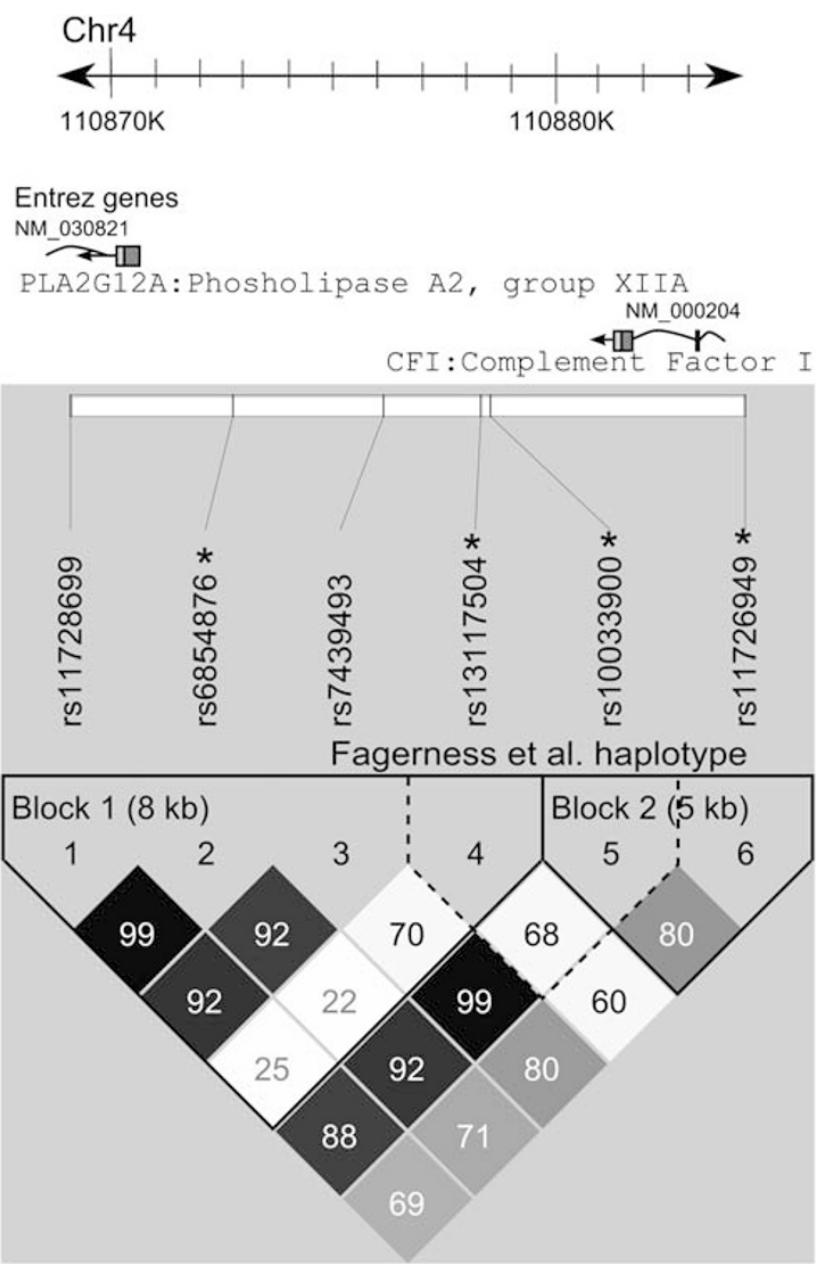

Figure 1 The $\mathrm{CFI}$ gene with relative positions of genotyped SNPs and haploview $^{6}$ LD plot. The CFI gene is transcribed from the negative strand of chromosome 4. Relative locations of the six SNPs genotyped in our study are shown. One SNP, rs11726949, occurs within an intron of $\mathrm{CFI}$. The remainder is found $3^{\prime}$ of the coding sequence with one SNP rs11728699 intronic to the adjacent PLA2G12A gene. Asterisks depict those SNPs also tested by Fagerness et al. The lower grid shows the two haploview-defined LD blocks (solid spine of LD with $D^{\prime}>0.8$ ) in solid lines and the haplotype examined by Fagnerness et al in dashed lines. 
Table 2 Single SNP association analysis

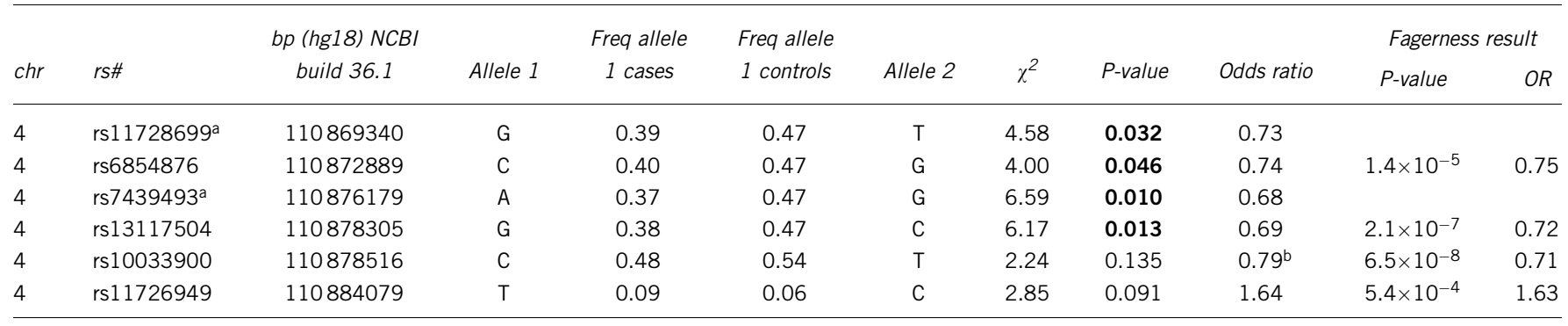

aNot genotyped in Fagerness et al. ${ }^{1}$

bodds ratio referent to $\mathrm{C}$ allele as per Fagerness et al. ${ }^{1}$

Bold type indicates significant results with a $P$-value $<0.05$

Table 3 Haplotype analyses of LD blocks identified using Haploview and Fagerness block

\begin{tabular}{|c|c|c|c|c|c|c|c|c|}
\hline \multirow[b]{2}{*}{ SNPS } & \multirow[b]{2}{*}{ Haplotype } & \multirow[b]{2}{*}{ Freq cases } & \multirow[b]{2}{*}{ Freq controls } & \multirow[b]{2}{*}{$\chi^{2}$} & \multirow[b]{2}{*}{$P$-value } & \multirow[b]{2}{*}{ Odds ratio } & \multicolumn{2}{|c|}{ Fagerness result } \\
\hline & & & & & & & $P$-value & Odds ratio \\
\hline Block 1 & GCAG & 0.36 & 0.45 & 6.05 & 0.014 & 0.69 & & \\
\hline \multirow{3}{*}{$\begin{array}{l}\text { rs11728699|rs6854876| } \\
\text { rs7439493|rs13117504 }\end{array}$} & TGGC & 0.59 & 0.51 & 3.89 & 0.048 & 1.34 & & \\
\hline & GCGC & 0.03 & 0.02 & 2.25 & 0.134 & 2.08 & & \\
\hline & TGAG & 0.02 & 0.02 & 0.01 & 0.919 & 0.94 & & \\
\hline Block 2 & TT & 0.08 & 0.04 & 5.26 & 0.022 & 2.15 & & \\
\hline \multirow[t]{3}{*}{ rs10033900|rs11726949 } & $\mathrm{CC}$ & 0.48 & 0.52 & 1.18 & 0.278 & 0.85 & & \\
\hline & CT & 0.01 & 0.02 & 0.64 & 0.425 & 0.61 & & \\
\hline & $\mathrm{TC}$ & 0.43 & 0.42 & 0.04 & 0.834 & 1.03 & & \\
\hline \multirow{4}{*}{$\begin{array}{l}\text { Fagerness et al haplotype } \\
\text { rs13117504|rs10033900 }\end{array}$} & GC & 0.35 & 0.43 & 4.56 & 0.033 & 0.72 & $4.39 \times 10^{-7}$ & 0.72 \\
\hline & $\mathrm{CT}$ & 0.48 & 0.42 & 3.32 & 0.069 & 1.31 & $1.18 \times 10^{-8}$ & 1.448 \\
\hline & $\mathrm{CC}$ & 0.13 & 0.11 & 0.99 & 0.319 & 1.25 & & \\
\hline & GT & 0.03 & 0.05 & 0.95 & 0.329 & 0.69 & & \\
\hline
\end{tabular}

Bold type indicates significant results with a $P$-value $<0.05$.

association between haplotypes and disease status (Table 3). For ease of comparison between studies, we also analysed the haplotype investigated by Fagerness et al (although this haplotype actually straddles the two blocks identified in our data). Two complementary haplotypes (GCAG and TGGC) account for approximately 95\% of chromosome variation across block 1 . Both are significantly associated with disease status in our data with the GCAG haplotype conferring protection $(\mathrm{OR}=0.69)$ and its complement TGGC conferring risk (OR 1.64). Haplotype 'TT' from block 2 is statistically significant $(P=0.02)$ with an OR of 2.15. This is of particular interest as this haplotype contains the most significant SNP identified by Fagerness et al, but neither of the SNPs reached formal significance when examined singularly within our sample. Similar to the single SNP analyses, the data from the two-SNP haplotype used by Fagerness et al are highly comparable between studies given the larger sample has much greater power.

Our results are based on an independent cohort ascertained from ophthalmic clinics in the Southampton region of the United Kingdom. Despite being based on a much smaller sample size, our data support the findings of Fagerness et al, implicating genomic variation in the region of the CFI gene with AMD disease susceptibility. These results enforce the suggestion that this region warrants comprehensive evaluation.

\section{ACKNOWLEDGEMENTS}

This work was supported by the Macula Vision Research Foundation and the Macular Disease Society.
Sarah Ennis ${ }^{1}$, Jane Gibson ${ }^{1}$, Angela J Cree ${ }^{2}$, Andrew Collins ${ }^{1}$ and Andrew J Lotery ${ }^{2,3}$

${ }^{1}$ Genetic Epidemiology and Bioinformatics Group, Human Genetics Division (Mp 808), Southampton General Hospital, University of Southampton, Southampton, UK; ${ }^{2}$ Clinical Neurosciences Division (Mp 806), Southampton General Hospital, University of Southampton, Southampton, UK; ${ }^{3}$ Southampton Eye Unit, Southampton General Hospital, Southampton, UK E-mail: s.ennis@southampton.ac.uk

1 Fagerness JA, Maller JB, Neale BM, Reynolds RC, Daly MJ, Seddon JM: Variation near complement factor I is associated with risk of advanced AMD. Eur J Hum Genet 2009; 17: $100-104$.

2 Klein RJ, Zeiss C, Chew EY et al: Complement factor $\mathrm{H}$ polymorphism in age-related macular degeneration. Science 2005; 308: 385-389.

3 Edwards AO, Ritter III R, Abel KJ, Manning A, Panhuysen C, Farrer LA: Complement factor $\mathrm{H}$ polymorphism and age-related macular degeneration. Science 2005; 308 : 421-424.

4 Haines JL, Hauser MA, Schmidt S et al: Complement factor $\mathrm{H}$ variant increases the risk of age-related macular degeneration. Science 2005; 308: 419-421.

5 Ennis S, Jomary C, Mullins R et al: Association between the SERPING1 gene and agerelated macular degeneration: a two-stage case-control study. Lancet 2008; 372: 1828-1834.

6 Barrett JC, Fry B, Maller J, Daly MJ: Haploview: analysis and visualization of LD and haplotype maps. Bioinformatics 2005; 21: 263-265.

7 Purcell S, Neale B, Todd-Brown K et al: PLINK: a tool set for whole-genome association and population-based linkage analyses. Am J Hum Genet 2007; 81: 559-575. 